\title{
A splicer that represses (translation)
}

\author{
Robin P. Wharton ${ }^{1}$ \\ Department of Molecular Genetics and Microbiology, Duke University, Durham, North Carolina 27710, USA
}

\begin{abstract}
Regulated translation and subcellular localization of maternal mRNAs underlies establishment of the antero-posterior axis in the Drosophila oocyte. In this issue of Genes \& Development, Besse et al. (pp. 195-207) show that a molecule better known as a regulator of alternative splicing in the nucleus, polypyrimidine tractbinding protein (PTB), is required for repression of oskar mRNA in the cytoplasm. Their work suggests that PTB need not engage oskar mRNA in the nucleus for efficient repression, providing an important counterexample to the increasingly popular idea that cytoplasmic regulation initiates in the nucleus.
\end{abstract}

mRNAs localized to the anterior and posterior poles of the oocyte encode the proteins that initiate a now wellunderstood cascade of gene regulation that patterns the Drosophila embryo (St Johnston 2005). Localization serves to restrict accumulation of the encoded proteins to a portion of the cytoplasm in the single large cell / $\sim 500$ $\mu \mathrm{m}$ long) that is the early, syncytial embryo. This strategy is widely employed in other cells that are either unusually large (the Xenopus oocyte) or elongated (neurons). A recent survey shows that many mRNAs in the Drosophila embryo exhibit subcellular localization, suggesting that the strategy may be more widely employed than suspected previously (Lecuyer et al. 2007).

If a mRNA is localized for the purpose of driving local protein accumulation, it must be repressed during transit. Studies of oskar (osk) mRNA, which is localized to the posterior pole of the oocyte, have illuminated the coupled regulation of translation and localization for many years. osk mRNA is synthesized in the accessory nurse cells of the egg chamber; a subset of the mRNA is transported in mRNPs to the posterior pole of the oocyte at stage 9 of oogenesis (Zimyanin et al. 2008). Only upon arrival at the posterior pole is the mRNA translated (Kim-Ha et al. 1991; Ephrussi and Lehmann 1992); the short encoded Osk isoform (Markussen et al. 1995) then nucleates assembly of germ line determinants that are incorporated into the primordial germ cells at the posterior pole.

Osk has a second essential role at the posterior poledirecting the localization and translational activation of nanos (nos) mRNA (Ephrussi and Lehmann 1992; Smith

[Keywords: Drosophila; PTB; oskar; translation; RNP assembly; hnRNP] ${ }^{1}$ Correspondence.

E-MAIL rwharton@duke.edu; FAX (919) 681-8984.

Article is online at http://www.genesdev.org/cgi/doi/10.1101/gad.1768509. et al. 1992). The resulting gradient of Nos protein patterns the posterior of the embryo, directing the formation of abdominal segments (Wharton and Struhl 1991). Although the mechanism by which Osk activates nos mRNA is unclear (and likely to be indirect), it is clear that Osk-dependent activation must be restricted to the posterior. Otherwise, the resulting generation of Nos in anterior regions of the embryo has lethal developmental consequences. Thus, osk mRNA must be repressed until its arrival at the posterior.

\section{The PTB splicing regulator as a repressor of osk translation}

In this issue of Genes \& Development, Ephrussi and colleagues (Besse et al. 2009) report the identification of a new factor required for repression of osk mRNA in the ovary-the polypyrimidine tract-binding protein (PTB). This factor has a rich, well-documented history as a regulator of alternative splicing in the nucleus and an activator of IRES-dependent translation in the cytoplasm. Reports that PTB can repress IRES-driven translation have relied primarily on overexpression experiments (at levels up to 10 -fold above endogenous) in cultured cells (Kim et al. 2000; Cornelis et al. 2005). The new study from Besse et al. (2009) is the strongest evidence to date that PTB represses translation in vivo.

Mammalian PTB binds to CU-rich elements in intronic or exonic silencer elements near alternative exons that are only sometimes included in the mature mRNA (Singh et al. 1995; Perez et al. 1997). PTB blocks exon definition and spliceosome assembly, although the precise mechanisms by which it does so are not yet clear (Wagner and Garcia-Blanco 2002; Rideau et al. 2006; Sharma et al. 2008). PTB has also been implicated as a core trans-acting factor for IRES-dependent translation of many viral and cellular messages (Sawicka et al. 2008), bypassing the requirement for a $\mathrm{m}^{7} \mathrm{GpppG}$ cap during infection and other cellular stress. In addition to these arenas, PTB has been implicated in a number of other mRNA transactions (Sawicka et al. 2008); its role in localization of $V g 1$ mRNA in the Xenopus oocyte (Cote et al. 1999) has been particularly influential in development of the idea that cytoplasmic mRNA regulation is initiated by events in the nucleus (a topic outlined later in this perspective). Drosophila PTB is closely related to the better studied human and frog proteins; but no direct demonstration that it plays a role in splicing regulation in flies has been 
reported. One way to address this issue would be to use microarrays that detect alternative splice isoforms to compare the transcriptome in wild-type and PTBknocked-down S2 cells, as described by Rio and colleagues (Blanchette et al. 2005).

PTB emerged from a screen for factors that colocalize with osk mRNA, as do the previously identified osk regulators Staufen, Bruno, and Hrp48 (Webster et al. 1997; Martin et al. 2003; Huynh et al. 2004; Yano et al. 2004). Besse et al. (2009) found an exon-trap insertion in the PTB locus (hephaestus in FlyBase but PTB in this paper) that encodes a GFP fusion that is localized to the oocyte posterior in stage 9 egg chambers, like wild-type osk. PTB-GFP (and PTB) were shown to track osk mRNA in experiments where either its production or localization were altered. Thus, PTB was a good candidate to regulate osk.

PTB is essential for Drosophila viability; to test for a role in osk regulation, Ephrussi and colleagues (Besse et al. 2009) therefore selectively ablated its function in the ovary by preparing germ line clones. Only hypomorphic alleles were found to support the development of egg chambers to the point where osk mRNA localization and repression can be usefully monitored. osk mRNA was mislocalized in about $20 \%$ of such PTB hypomorphic stage 9 egg chambers, although localization was essentially normal by stage 10 . This "recovery" was interpreted to result from a transient delay in reorganization of the microtubule cytoskeleton, which plays a key role in mediating transportation of osk mRNA (Zimyanin et al. 2008). These observations paint a picture in which PTB plays a relatively minor and indirect role in osk localization in contrast to its central role in the localization of $V g 1$ mRNA in Xenopus oocytes. Whether this represents a fundamental difference between the two systems, or residual PTB activity in the ovaries studied by Besse et al. (2009) remains to be seen.

A compelling role for PTB in the repression of osk mRNA emerged from examination of Osk protein in the same germ line clones. Unlike wild-type egg chambers, in which Osk is found no earlier than late stage 8 and then only at the posterior pole of the oocyte, Osk was shown to accumulate both prematurely and in inappropriate (e.g., more anterior) regions of the oocyte in over half of the PTB mutant egg chambers examined. Similar ectopic accumulation of Osk had been observed upon mutating the Bru-binding sites in the osk 3' untranslated region (UTR) (Kim-Ha et al. 1995).

Besse et al. (2009) next addressed the question of whether PTB acts directly on osk mRNA to repress its translation. A priori, indirect regulation was a reasonable possibility, particularly if $\mathrm{PTB}$, in fact, proves to regulate alternative splicing of as many pre-mRNAs as does Hrp48 (for example, Blanchette et al. 2005). However, Besse et al. (2009) found that the endogenous PTB in ovarian extracts binds preferentially to the osk 3' UTR shown previously to be required for translational repression and localization. Unlike repressors of other maternal mRNAs that act via one or two discrete sites in target mRNAs (e.g., Smaug or Pumilio) (Wharton and Struhl 1991; Smibert et al. 1996), PTB seemed likely to act via a different mechanism, based on two observations. First, the 1055 nt osk 3'
UTR was found to be littered with evolutionarily conserved CU-rich sequences similar to PTB-binding sites in well-characterized mammalian splicing silencers (Singh et al. 1995; Perez et al. 1997). Second, subdivision of the 3' UTR revealed that each of the five distinct fragments tested could bind PTB. As binding specificity controls, neither a protein-coding region of osk mRNA nor the Y14 mRNA bound appreciably to PTB; and only the osk 3' UTR fragments with previously characterized Bruno-binding sites bound Bruno. Thus, PTB likely binds in vivo to multiple sites in the osk 3' UTR.

To investigate PTB binding in vitro, Besse et al. (2009) purified from bacteria a PTB fusion to the monomeric MBP carrier protein and used it in gel mobility shift experiments. These revealed selective binding to the osk $5^{\prime}$ and $3^{\prime}$ UTRs, both of which have been implicated in translational control (Gunkel et al. 1998). However, the mode of binding to each UTR appears to be different: PTB forms two discrete high-mobility complexes on the 5' UTR, and a high-order aggregate (containing an unknown number of proteins) that barely enters the gel on the $3^{\prime}$ UTR. How might this aggregate arise? Mammalian PTB has four RNA recognition motif (RRM)-binding motifs, each capable of binding a CU-rich element (Oberstrass et al. 2005). In addition, RRM3 and RRM4 have been shown to interact with each other such that, if each is bound to a CU-rich element within a single RNA molecule, a protein cross-linked RNA loop would be formed. If the fly protein has similar properties, then either inter- or intramolecular PTB-bridged cross-links might account for the low mobility complexes observed in the experiments of Ephrussi and colleagues (Besse et al. 2009).

Besse et al. (2009) then wondered whether PTB might drive aggregation of osk mRNAs in vivo much as it does when incubated with the 3' UTR in vitro. Previous work had shown that reporter mRNAs bearing the osk 3' UTR are efficiently localized to the oocyte posterior, but only if allowed to hitchhike on endogenous osk mRNPs (Hachet and Ephrussi 2004). Strikingly, Ephrussi and colleagues (Besse et al. 2009) found that hitchhiking of an EGFP reporter mRNA bearing the osk 3' UTR is dependent on normal PTB activity, consistent with the idea that PTBmediated cross-linking drives the formation of mRNP aggregates in vivo. Further support for this idea came from the observation that osk mRNP transport particles in the center of stage 9 oocytes are about one-third normal size, as measured by high-resolution in situ hybridization and immunoelecton microscopy. It would be interesting to know whether, at later stages when osk localization has recovered, the osk mRNPs are still diminuitive. But, taken with the ability of PTB to promote hitchhiking and the derepression of osk in PTB mutants, Besse et al. (2009) concluded that PTB may repress osk by sequestering it from ribosomes in large mRNP aggregates.

The results outlined above raise further questions about the role of PTB in osk regulation. Here, I focus on two topics. First, if repression and/or localization is mediated by PTB-dependent cross-linking into high-order aggregates, might concatemerized PTB-binding sites suffice to drive these events (or to allow hitchhiking of a reporter 
mRNA on the native osk mRNP)? Oligomerized PTBbinding sites are sufficient to confer IRES activity in mammalian cells (Mitchell et al. 2005), establishing a precedent for this sort of approach. Second, what are the relative contributions of Bruno, Hrp48, and PTB toward repressing osk? Are the weak developmental phenotypes revealed by genetic analysis (Webster et al. 1997; Yano et al. 2004) due to redundancy? Or to the residual activity in partial loss-of-function backgrounds? Or perhaps due to sequential repressor action (much as the responsibility for repression of nos mRNA passes from Glorund to Smg; Kalifa et al. 2006), such that early failures in repression are masked by later successes? With the currently available alleles, this set of questions will be difficult to answer, as it is not possible to eliminate function of any one of the three factors and study the regulation of osk that occurs during late stages of oogenesis. Ablation of the Bruno-binding sites in cis appears to cause much stronger derepression than does a reduction in activity of any of the three trans-acting factors (Kim-Ha et al. 1995). However, some of the Bruno- and Hrp48binding sites are close to each other and some overlap (Yano et al. 2004), raising the possibility that the mutations studied affect binding of both factors to the osk 3' UTR. Perhaps these issues can be addressed in vitro by selectively depleting each factor from the ovarian extracts that support regulation of osk mRNA.

\section{Other dual function regulators of splicing and translation in Drosophila}

Many RNA-binding proteins shuttle between the nucleus and cytoplasm, even if they prefer to reside in one of the compartments. Is it surprising, then, that PTB has dual roles in regulating splicing and translation? The simple answer is no- of the other well-characterized repressors of posteriorly localized mRNAs (osk and nos), one certainly plays a direct role in splicing and two others probably do. The known or suspected dual function regulators all have multiple RRMs, versatile motifs for RNA recognition and protein-protein interaction.

Of the other osk repressors, Hrp48 is better known for its role in splicing regulation than translation. Rio and colleagues (Blanchette et al. 2005) have studied its function in regulation of the splicing of P-element transposase pre-mRNA in some detail. They concluded recently that it regulates at least 90 pre-mRNAs, some in collaboration with the PSI cofactor critical for transposase splicing and some not (Blanchette et al. 2005). Drosophila Bruno has not yet been shown to play a role in splicing regulation. But the protein appears to shuttle (Snee et al. 2008) and mammalian orthologs have been implicated in splicing (Suzuki et al. 2002).

Of the two well-characterized nos repressors, Glo (the Drosophila hnRNP F/H homolog) has been shown to interact directly with Hrp48 and the splicing factor Halfpint in vitro, consistent with observations that mammalian hnRNP $\mathrm{H}$ is involved in regulation of alternative splicing (Kalifa et al. 2008). Only Smg seems to steer clear of the nucleus, sticking to the cytoplasm where its main role is to direct the degradation of a large subset of maternal mRNAs during the transition from maternal to zygotic control of development (Tadros et al. 2007).

In summary, dual function may become the rule rather than the exception, particularly as sophisticated genetic tools reveal functions for essential RNA-binding proteins in repression (and localization).

\section{Is the nuclear experience overrated?}

The idea that cytoplasmic mRNA transactions are obligatorily set up in the nucleus has become generally accepted (Giorgi and Moore 2007), despite documented exceptions (perhaps most notably, the apical localization of various mRNAs microinjected into the cytoplasm of the Drosophila embryo) (Bullock and Ish-Horowicz 2001; Wilkie and Davis 2001). One broad reason for the wave of enthusiasm has been the demonstration that nonsense mediated decay (NMD) in the cytoplasm (or at the nuclear/cytoplasmic boundary during a pioneer round of translation) is initiated by deposition of the exon junction complex (EJC) during splicing. Following is a brief summary of two additional influential contributions: the discovery that the EJC is required for osk localization in Drosophila, and isomerization of the $V g 1 \mathrm{mRNP}$ during its localization in the Xenopus oocyte.

Genetic experiments revealed that osk mRNA localization requires factors that proved to be core components of the EJC (Hachet and Ephrussi 2001; Palacios et al. 2004). These observations were initially somewhat puzzling, since the EJC is thought to be deposited on every spliced mRNA, even though very few of these are localized. Subsequent experiments showed that splicing of osk pre-mRNA is essential for its localization, and that the position of the first intron is critical, presumably for the deposition of an EJC at a privileged position where it facilitates the assembly of a localization-competent RNP (Hachet and Ephrussi 2004).

Work on the localization of $V g 1$ mRNA to the vegetal pole of the Xenopus oocyte has led to a convincing argument by Mowry and colleagues (Kress et al. 2004) that its cytoplasmic fate is determined by earlier events in the nucleus. Localization of $V g 1$ is dependent on binding of two factors to a cis-acting element in the $3^{\prime}$ UTR-PTB and Vg1RBP/Vera (Deshler et al. 1998; Cote et al. 1999). Upon immunoprecipitation of separated nuclear and cytoplasmic fractions, both proteins are found in association with each other and with the mRNA (Kress et al. 2004). However, although Vg1RBP/Vera is part of the nuclear mRNP, it is not directly bound to the RNA until the complex is exported to the cytoplasm. Direct binding of Vg1RBP/Vera (assayed by UV crosslinking) is dependent on PTB binding, presumably a prior "good" act committed in the nucleus (Lewis et al. 2008).

In light of the nuclear role of Xenopus PTB in subsequent cytoplasmic trafficking, Besse et al. (2009) asked whether PTB must engage osk mRNA in the nucleus to establish a cytoplasmic repression complex in Drosophila. The experimental approach was to express a nuclear localization signal (NLS)-deleted PTB derivative in PTB 
mutant egg chambers, assaying osk mRNA localization and translational repression as described above. Somewhat surprisingly, expression of a fusion of either wildtype PTB or the $\triangle$ NLS derivative to GFP restored translational repression. The $\Delta$ NLS derivative appeared to be effectively excluded from the nucleus, since it fails to repress accumulation of the endogenous protein, a welldocumented autoregulatory activity of mammalian PTB (Wollerton et al. 2004). (This observation further increases the liklihood that the fly and mammalian proteins have very similar nuclear functions, since autoregulation is achieved by controlling the splicing of PTB pre-mRNA.) The $\Delta$ NLS protein also rescued the minor osk localization defects in mutant egg chambers, albeit somewhat less efficiently than did wild-type PTB, suggesting that nuclear engagement may be important for localization.

Nevertheless, a major conclusion from the work of Besse et al. (2009) is that nuclear recruitment of PTB is not required for cytoplasmic repression in Drosophila, with the caveat that residual wild-type PTB in the hypomorphic background employed (of necessity) might initiate the process in the nucleus. Perhaps an attractive parallel with the action of PTB on $V g 1$ mRNA first in the nucleus and then in the cytoplasm of the Xenopus oocyte will yet carry the day.

\section{Is formation of large mRNPs particles/aggregates an essential aspect of repression or an epiphenomenon?}

Many RNA transactions occur in large assemblies: mRNA is translated on ribosomes, degraded in P bodies, and localized in particles large enough to be visible by light microscopy. Might an essential aspect of translational repression occur by sequestration in large aggregates/particles that exclude ribosomes?

The properties of PTB reported by Besse et al. (2009) make an attractive correlative case for the importance of particle formation. In vitro, PTB forms high-order aggregates specifically on the osk 3' UTR; in vivo, it is required for translational repression and promotes hitchhiking of mRNAs bearing the osk 3' UTR to the posterior pole. Strengthening the argument will likely require the isolation of mutants that selectively interfere with oligomeric binding to the osk 3' UTR (and not, for example, formation of discrete complexes on the osk 5' UTR). Such an approach is undoubtedly easier to propose than carry out, particularly on a molecule with four RNA-binding domains.

Two other examples of large, repressed Drosophila mRNP particles have been reported. First, binding sites for Bru (Bru reponse elements or BREs) were shown (1) to promote the formation of fast-sedimenting mRNP aggregates or particles, and (2) to mediate cap-independent repression (Chekulaeva et al. 2006). The BREs also were shown to mediate formation of cross-linked complexes containing multiple RNA species in ovarian extracts (e.g., RNA hitchhiking). Taken together, the experiments suggested a model in which Bru cross-links mRNAs into large silencing aggregates/particles as part of its repressive activity. However, Besse et al. (2009) report that the BREs are neither necessary nor sufficient for hitchhiking of mRNAs bearing the osk 3' UTR in vivo. Perhaps in vitro Bru can promote aggregation by binding to BREs, whereas in vivo Bru mediates protein-protein interactions that stabilize aggregates primarily cross-linked by PTB.

A second report of large, silenced mRNP particles involves miR2-dependent repression in embryonic extracts (Thermann and Hentze 2007). The mechanism by which micro-RNAs inhibit translation has been somewhat controversial; the experiments of Thermann and Hentze (2007) demonstrated that miR2 acts by blocking formation of the $48 \mathrm{~S}$ initiation complex. In the course of experiments to resolve both $48 \mathrm{~S}$ pre- and $80 \mathrm{~S}$ postinitiation complexes, they made the surprising observation that miR2 promotes the formation of heavy, puromycin-insensitive but EDTA-sensitive aggregates that they named "pseudopolysomes." The relevance of these aggregates to repression is not clear, since miR2 promoted their formation even on a substrate refractory to repression by virtue of possession of an ApppG 5' cap.

In summary, it seems likely that PTB will be important for at least one step in the assembly of silenced osk mRNP particles, given its role in mediating mRNA hitchhiking in vivo. Future work is likely to focus on the identification of other components of these particles and efforts to pin down the mechanism by which they block translation.

\section{Acknowledgments}

I thank Maki Asano and Krystle Nomie for reading the manuscript.

\section{References}

Besse, F., Lopez de Quinto, S., Marchand, V., Trucco, A., and Eprhussi, A. 2009. Drosophila PTB promotes formation of high-order RNP particles and repressses oskar translation. Genes \& Dev. (this issue). doi: 10.1101/gad.505709.

Blanchette, M., Green, R.E., Brenner, S.E., and Rio, D.C. 2005. Global analysis of positive and negative pre-mRNA splicing regulators in Drosophila. Genes \& Dev. 19: 1306-1314.

Bullock, S.L. and Ish-Horowicz, D. 2001. Conserved signals and machinery for RNA transport in Drosophila oogenesis and embryogenesis. Nature 414: 611-616.

Chekulaeva, M., Hentze, M.W., and Ephrussi, A. 2006. Bruno acts as a dual repressor of oskar translation, promoting mRNA oligomerization and formation of silencing particles. Cell 124: 521-533.

Cornelis, S., Tinton, S.A., Schepens, B., Bruynooghe, Y., and Beyaert, R. 2005. UNR translation can be driven by an IRES element that is negatively regulated by polypyrimidine tract binding protein. Nucleic Acids Res. 33: 3095-3108.

Cote, C.A., Gautreau, D., Denegre, J.M., Kress, T.L., Terry, N.A., and Mowry, K.L. 1999. A Xenopus protein related to hnRNP I has a role in cytoplasmic RNA localization. Mol. Cell 4: 431-437.

Deshler, J.O., Highett, M.I., Abramson, T., and Schnapp, B.J. 1998. A highly conserved RNA-binding protein for cytoplasmic mRNA localization in vertebrates. Curr. Biol. 8: 489-496.

Ephrussi, A. and Lehmann, R. 1992. Induction of germ cell formation by oskar. Nature 358: 387-392.

Giorgi, C. and Moore, M.J. 2007. The nuclear nurture and cytoplasmic nature of localized mRNPs. Semin. Cell Dev. Biol. 18: 186-193. 
Gunkel, N., Yano, T., Markussen, F.H., Olsen, L.C., and Ephrussi, A. 1998. Localization-dependent translation requires a functional interaction between the $5^{\prime}$ and $3^{\prime}$ ends of oskar mRNA. Genes \& Dev. 12: 1652-1664.

Hachet, O. and Ephrussi, A. 2001. Drosophila Y14 shuttles to the posterior of the oocyte and is required for oskar mRNA transport. Curr. Biol. 11: 1666-1674.

Hachet, O. and Ephrussi, A. 2004. Splicing of oskar RNA in the nucleus is coupled to its cytoplasmic localization. Nature 428: 959-963.

Huynh, J.R., Munro, T.P., Smith-Litiere, K., Lepesant, J.A., and St Johnston, D. 2004. The Drosophila hnRNPA/B homolog, Hrp48, is specifically required for a distinct step in osk mRNA localization. Dev. Cell 6: 625-635.

Kalifa, Y., Huang, T., Rosen, L.N., Chatterjee, S., and Gavis, E.R. 2006. Glorund, a Drosophila hnRNP F/H homolog, is an ovarian repressor of nanos translation. Dev. Cell 10: 291-301.

Kalifa, Y., Armenti, S.T., and Gavis, E.R. 2008. Glorund interactions in the regulation of gurken and oskar mRNAs. Dev. Biol. doi: 10.1016/j.ydbio.2008.10.032.

Kim, Y.K., Hahm, B., and Jang, S.K. 2000. Polypyrimidine tractbinding protein inhibits translation of bip mRNA. I. Mol. Biol. 304: 119-133.

Kim-Ha, J., Smith, J.L., and Macdonald, P.M. 1991. oskar mRNA is localized to the posterior pole of the Drosophila oocyte. Cell 66: 23-35.

Kim-Ha, J., Kerr, K., and Macdonald, P.M. 1995. Translational regulation of oskar mRNA by Bruno, an ovarian RNAbinding protein, is essential. Cell 81: 403-412.

Kress, T.L., Yoon, Y.J., and Mowry, K.L. 2004. Nuclear RNP complex assembly initiates cytoplasmic RNA localization. $J$. Cell Biol. 165: 203-211.

Lecuyer, E., Yoshida, H., Parthasarathy, N., Alm, C., Babak, T., Cerovina, T., Hughes, T.R., Tomancak, P., and Krause, H.M. 2007. Global analysis of mRNA localization reveals a prominent role in organizing cellular architecture and function. Cell 131: 174-187.

Lewis, R.A., Gagnon, J.A., and Mowry, K.L. 2008. PTB/hnRNP I is required for RNP remodeling during RNA localization in Xenopus oocytes. Mol. Cell. Biol. 28: 678-686.

Markussen, F.H., Michon, A.M., Breitwieser, W., and Ephrussi, A. 1995. Translational control of oskar generates short OSK, the isoform that induces pole plasm assembly. Development 121: 3723-3732.

Martin, S.G., Leclerc, V., Smith-Litiere, K., and St Johnston, D. 2003. The identification of novel genes required for Drosophila anteroposterior axis formation in a germline clone screen using GFP-Staufen. Development 130: 4201-4215.

Mitchell, S.A., Spriggs, K.A., Bushell, M., Evans, J.R., Stoneley, M., Le Quesne, J.P., Spriggs, R.V., and Willis, A.E. 2005. Identification of a motif that mediates polypyrimidine tractbinding protein-dependent internal ribosome entry. Genes \& Dev. 19: 1556-1571.

Oberstrass, F.C., Auweter, S.D., Erat, M., Hargous, Y., Henning, A., Wenter, P., Reymond, L., Amir-Ahmady, B., Pitsch, S., Black, D.L., et al. 2005. Structure of PTB bound to RNA: Specific binding and implications for splicing regulation. Science 309: 2054-2057.

Palacios, I.M., Gatfield, D., St Johnston, D., and Izaurralde, E. 2004. An eIF4AIII-containing complex required for mRNA localization and nonsense-mediated mRNA decay. Nature 427: 753-757.

Perez, I., Lin, C.H., McAfee, J.G., and Patton, J.G. 1997. Mutation of PTB binding sites causes misregulation of alternative 3 ' splice site selection in vivo. RNA 3: 764-778.
Rideau, A.P., Gooding, C., Simpson, P.J., Monie, T.P., Lorenz, M., Huttelmaier, S., Singer, R.H., Matthews, S., Curry, S., and Smith, C.W. 2006. A peptide motif in Raverl mediates splicing repression by interaction with the PTB RRM2 domain. Nat. Struct. Mol. Biol. 13: 839-848.

Sawicka, K., Bushell, M., Spriggs, K.A., and Willis, A.E. 2008. Polypyrimidine-tract-binding protein: A multifunctional RNA-binding protein. Biochem. Soc. Trans. 36: 641-647.

Sharma, S., Kohlstaedt, L.A., Damianov, A., Rio, D.C., and Black, D.L. 2008. Polypyrimidine tract binding protein controls the transition from exon definition to an intron defined spliceosome. Nat. Struct. Mol. Biol. 15: 183-191.

Singh, R., Valcarcel, J., and Green, M.R. 1995. Distinct binding specificities and functions of higher eukaryotic polypyrimidine tract-binding proteins. Science 268: 1173-1176.

Smibert, C.A., Wilson, J.E., Kerr, K., and Macdonald, P.M. 1996. smaug protein represses translation of unlocalized nanos mRNA in the Drosophila embryo. Genes \& Dev. 10: 26002609.

Smith, J.L., Wilson, J.E., and Macdonald, P.M. 1992. Overexpression of oskar directs ectopic activation of nanos and presumptive pole cell formation in Drosophila embryos. Cell 70: 849-859.

Snee, M., Benz, D., Jen, J., and Macdonald, P.M. 2008. Two distinct domains of Bruno bind specifically to the oskar mRNA. RNA Biol. 5: 1-9.

St Johnston, D. 2005. Moving messages: The intracellular localization of mRNAs. Nat. Rev. Mol. Cell Biol. 6: 363-375.

Suzuki, H., Jin, Y., Otani, H., Yasuda, K., and Inoue, K. 2002. Regulation of alternative splicing of $\alpha$-actinin transcript by Bruno-like proteins. Genes Cells 7: 133-141.

Tadros, W., Goldman, A.L., Babak, T., Menzies, F., Vardy, L., OrrWeaver, T., Hughes, T.R., Westwood, J.T., Smibert, C.A., and Lipshitz, H.D. 2007. SMAUG is a major regulator of maternal mRNA destabilization in Drosophila and its translation is activated by the PAN GU kinase. Dev. Cell 12: 143-155.

Thermann, R. and Hentze, M.W. 2007. Drosophila miR2 induces pseudo-polysomes and inhibits translation initiation. Nature 447: 875-878.

Wagner, E.J. and Garcia-Blanco, M.A. 2002. RNAi-mediated PTB depletion leads to enhanced exon definition. Mol. Cell 10: 943-949.

Webster, P.J., Liang, L., Berg, C.A., Lasko, P., and Macdonald, P.M. 1997. Translational repressor bruno plays multiple roles in development and is widely conserved. Genes \& Dev. 11: 2510-2521.

Wharton, R.P. and Struhl, G. 1991. RNA regulatory elements mediate control of Drosophila body pattern by the posterior morphogen nanos. Cell 67: 955-967.

Wilkie, G.S. and Davis, I. 2001. Drosophila wingless and pairrule transcripts localize apically by dynein-mediated transport of RNA particles. Cell 105: 209-219.

Wollerton, M.C., Gooding, C., Wagner, E.J., Garcia-Blanco, M.A., and Smith, C.W. 2004. Autoregulation of polypyrimidine tract binding protein by alternative splicing leading to nonsensemediated decay. Mol. Cell 13: 91-100.

Yano, T., Lopez de Quinto, S., Matsui, Y., Shevchenko, A., and Ephrussi, A. 2004. Hrp48, a Drosophila hnRNPA/B homolog, binds and regulates translation of oskar mRNA. Dev. Cell 6: 637-648.

Zimyanin, V.L., Belaya, K., Pecreaux, J., Gilchrist, M.J., Clark, A., Davis, I., and St Johnston, D. 2008. In vivo imaging of oskar mRNA transport reveals the mechanism of posterior localization. Cell 134: 843-853. 


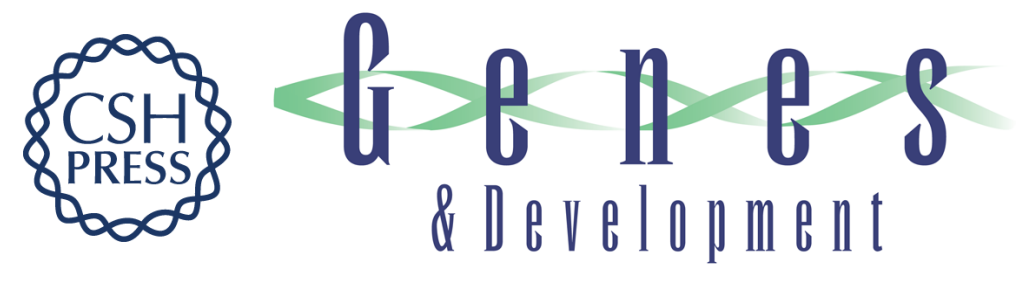

\section{A splicer that represses (translation)}

Robin P. Wharton

Genes Dev. 2009, 23:

Access the most recent version at doi:10.1101/gad.1768509

\section{Related Content Drosophila PTB promotes formation of high-order RNP particles and represses oskar translation \\ Florence Besse, Sonia López de Quinto, Virginie Marchand, et al. \\ Genes Dev. January, 2009 23: 195-207 \\ References This article cites 44 articles, 13 of which can be accessed free at: \\ http://genesdev.cshlp.org/content/23/2/133.full.html\#ref-list-1 \\ Articles cited in: \\ http://genesdev.cshlp.org/content/23/2/133.full.html\#related-urls \\ License \\ Email Alerting Receive free email alerts when new articles cite this article - sign up in the box at the top \\ Service right corner of the article or click here.}

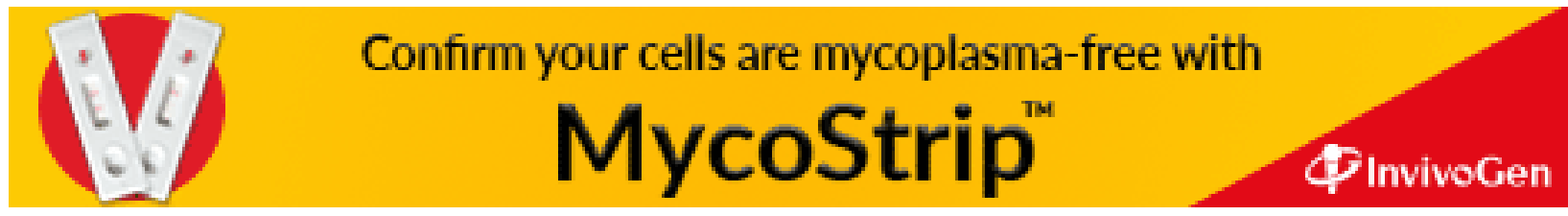

